\title{
Proteomics Analysis and Identification of Critical Proteins and Network Interactions That Regulate the Specific Deposition of IMF of Jingyuan Chicken
}

\section{Zengwen Huang}

Ningxia University https://orcid.org/0000-0002-7223-4047

Juan Zhang

Ningxia University

Yaling Gu ( $\square$ guyaling@sina.com )

Ningxia University

Guosheng Xin

Ningxia University

Zhengyun Cai

Ningxia University

Xianfeng Yin

Neijiang Vocational and Technical College

Xiaofang Feng

Ningxia University

Tong Mu

Ningxia University

Chaoyun Yang

Ningxia University

Research article

Keywords: Jingyuan chicken, Leg muscle, Breast muscle, Meat quality, Proteomics, IMF

Posted Date: August 24th, 2020

DOI: https://doi.org/10.21203/rs.3.rs-54060/v1

License: (1) (i) This work is licensed under a Creative Commons Attribution 4.0 International License.

Read Full License 


\section{Abstract}

Background:Improving the production efficiency and quality of broiler chickens has become a hot topic in molecular breeding of the broiler industry. There are several factors, among which intramuscular fat (IMF) content is one of the essential factors determining chicken quality. The chicken carcass weight of $40-50 \%$ consisted of the leg muscles and breast muscles, and the research on chicken meat quality was performed on that part. Jingyuan chicken is one of the high-quality local chicken species in China. However, the molecular mechanism of IMF specific deposition in different parts of Jingyuan chicken is still unclear. In order to screen and identify the proteins that regulate IMF specific deposition in different parts of Jingyuan chicken and analyze network interaction, the present study selected 180-day-old Jingyuan chicken as the research material, using proteomic techniques and PRM experimental methods to carry out basic research on leg muscle and breast muscle.

Results:Three key signaling pathways and ten essential functional proteins regulating IMF specific deposition of Jingyuan chicken were identified, while the molecular mechanism of the deposition of proteins was explained, and analyzed the network interaction between these key functional proteins.

Conclusions:The results of this study are of great scientific value and theoretical significance not only for improving chicken quality but also for molecular breeding of high-quality local chickens.

\section{Background}

Poultry meat has become the second-largest meat consumption in China, and its demand is increasing year by year, which shows that poultry meat has become an essential item to human beings (Dengfei et al. 2016). The growth of poultry production has been outstanding due to the remarkable achievements made by researchers through genetic improvement techniques on the growth rate, feed remuneration, and muscle yield of poultry (Laura et al. 2017; Zhichao et al. 2018). Unfortunately, it is regrettable that the quality of poultry meat has continued to decline as a result of the development in poultry production and the constant growth in muscle production (Muazzez et al. 2016). With the continuous improvement of the human dietary structure, the demand for meat products has gradually changed from quantity to quality, in particular the quality and flavor of poultry meat. Therefore, improving both the quality and quantity of broilers has become a hot topic in the current research. Chicken yield and meat quality traits are closely related to living body weight, carcass weight and other characteristics, of which between 40 and 50 percent of carcass weight is composed of leg and breast muscles (Chen Gangyao et al. 2012), Therefore, to improve the quality of chicken one should concentrate on breast and leg muscles of chicken. It has been found that there are significant differences in meat quality between leg muscle and breast muscle. Furthermore, several other parameters, including $\mathrm{pH}$, water capacity, cooking loss, muscle fiber, intramuscular fat (IMF) content, and inosinic acid, are responsible for determining the quality of meat (Chen Jilan et al. 2005). 
IMF refers to the fat distributed among muscle fibers in muscle tissue. The content of IMF plays a key role in the flavor, freshness, juiciness, taste, tenderness, and color of meat (MA et al. 2015). Studies have shown that IMF is derived from adipocytes between the myofascial membrane and myointimal, and these fat cells are derived from myogenic PDGFRa+ progenitor cells (Guan et al. 2017; Wang et al. 2017) and MyoD-directed differentiation of Myf5- and Pax3-non-myogenic progenitor cells (Liu et al. 2012).

Previous studies have suggested that the IMF content can be determined by both chronologically (Lin Yaqiu et al. 2015), and heritability ( $\mathrm{H} 2=0.5)$ (Liu et al. 2017). The studies on the molecular mechanism of chicken IMF specific deposition are exhibited into four aspects which are as follows:

(i) The biochemical mechanism of IMF affecting meat flavor was analyzed by previous researchers (Liu et al. 2017).

(ii) Considering the dimension of development, we have explored the deposition rules of IMF in vivo and the primary functional genes and signal pathways regulated at various stages. For example, it was found that the late embryonic stage is also one of the critical periods for intramuscular fat deposition. Studies have identified the essential roles of PPAR, fatty acid oxidation, cell connectivity, and other related pathways in intramuscular fat deposition (Zhang et al. 2017).

(iii) The fat deposition on different parts of the body roughly elaborated the molecular regulatory mechanism for differential fat deposition in breasts, leg muscle, and abdominal fat was compared with each other (Cui et al. 2018).

(iv) It is confirmed that genomic breeding methods have some potential in improving intramuscular fat deposition and meat quality (Jiang et al., .2017; Cui et al., .2018; Liu et al., .2019).

But in general, the regulation of IMF is the product of the interaction of multiple signaling molecules. The current research on IMF deposition mostly remains at the genetic level and the difference in content between varieties. However, the regulation of IMF specific deposition for critical proteins in different parts are not available as few related studies on molecular mechanisms and interactions between proteins are obtained so far.

China has abundant high-quality local broiler germplasm resources, with delicious meat, high tolerance of disease, and strong adaptability. Jingyuan chicken is one of the five district-level livestock and poultry genetic resources in the Ningxia Autonomous Region, and it is also an outstanding broiler breed in the Animal and Poultry Genetic Resources from China. After doing a lot of basic research on the production performance and meat quality of Jingyuan chicken, we found that the IMF content of Jingyuan chicken was higher than that of other breeds and significantly greater than that of fast large-scale broiler AA chicken. This biological characteristic is consistent with the results of a comparative study on the intramuscular fat deposition of other local broilers and AA Broilers by many researchers. Besides, Jingyuan chicken also contains certain unsaturated fatty acids (eicosapentaenoic acid, docosapentaenoic acid, 32-carbohexaenoic acid, etc.) that have exceptional nutritional value for the 
human body. However, the mechanism of the specific deposition of IMF in different parts of Jingyuan chicken is not clear. The particular expression and regulation of proteins in its body and the network of interactions need to be deeply analyzed.

In summary, to improve chicken quality and production performance, in particular, increasing the IMF content in chicken, has become a hot topic in current research. IMF deposition is influenced not only by spatiotemporal but also by heritability. The occurrence, development, and deposition of IMF cells are extremely complex, including an interactive physiological and biochemical process. It is difficult to pass a gene, protein expression pattern, or a signal transduction pathway to clarify the mechanism of IMF specific deposition. Nonetheless, the effect and control of genetic, environmental, and nutritional factors on animal intramuscular fat deposition are inseparable from the involvement of main functional proteins and thereby associated signaling networks in the animal body. Although the animal molecular nutrition pathway can also regulate lipid metabolism and intramuscular fat deposition, it is ultimately the result of specific protein expression and translation modification (Li et al. 2016, 2016; Wang et al. 2016, 2017). Therefore, the use of proteomics technology to screen and identify essential proteins and signal network pathways that control IMF specific deposition in different parts of the chicken, providing relevant molecular data for further improvement of chicken quality and accurate broiler molecular breeding.

Based on the previous research of the research group, we selected the high-quality local broiler breedJingyuan chicken as the research object. We used the label-free proteomics technique and parallel reaction monitoring method (PRM) to screen and identify the critical functional proteins that regulate the specific deposition IMF the leg muscle and breast muscle of Jingyuan chicken. The results have not only significant biological and economic value to improve the flavor and quality of chicken but also have typical representative significance to the molecular breeding research of high-quality local broilers.

\section{Methods}

\section{Reagent}

Sequencing grade modified trypsin has been obtained from Promega (Fitchburg, WI). lodoacetamide (IAA), DTT, TFA, EDTA, urea, tetraethylammonium borohydride (TEAB), 2-DQuantkit, etc. have all been purchased from Sigma (St. Louis, MO) and GE Healthcare (Buckinghamshire, United Kingdom). Besides, protease inhibitor cocktail III, TMT kit, ACN, purified water, formic acid (FA), etc. were procured from Thermo Fisher Scientific (Waltham, MA) and German Fluke (Buches, Germany) companies.

\section{Animals and samples}

In this research, 30 (hens) of 180-day old white feather Jingyuan chicken with an average live weight of $2.5 \pm 0.23 \mathrm{~kg}$ were selected from the breeding center of Chaona chicken in Pengyang County of Ningxia Hui Autonomous Region. Their muscles of the leg and breasts were collected in an aseptic environment and stored at $-80^{\circ} \mathrm{C}$ for future use. 
In accordance with the "Environmental Protection Law of the People's Republic of China" and the "Animal Epidemic Prevention Law of the People's Republic of China", we burn and bury animal carcasses after experiments to prevent them from causing environmental pollution and harm to human health.

\section{Protein extraction and enzymatic hydrolysis}

The sample was removed from the $-80^{\circ} \mathrm{C}$ refrigerator and ground it thoroughly in a mortar with liquid nitrogen. After grinding into powder, four times volume lysis buffer (8M urea, $1 \%$ protease inhibitor) was added for ultrasonic lysis. Then centrifuged of $12000 \mathrm{~g}$ at $4^{\circ} \mathrm{C}$ for $10 \mathrm{~min}$, cell debris was removed, and the protein concentration was determined by the BCA kit. Upon dilution of the lysed proteins with dithiothreitol and iodoacetamide, trypsin was added at a mass ratio of 1:50 (trypsin: protein), and enzymatic hydrolysis was performed overnight at $37^{\circ} \mathrm{C}$. Then trypsin was then added at 1:100 mass ratio (trypsin: protein) to continue enzymatic hydrolysis for $4 \mathrm{~h}$.

\section{Liquid chromatography-mass spectrometry analysis}

The peptide was dissolved in an aqueous solution containing $0.1 \%$ formic acid and acetonitrile solution containing $0.1 \%$ formic acid by liquid chromatography mobile phase $A$ and mobile phase $B$, respectively. Then peptide was separated by Nano ultra-high performance liquid system and injected into capillary ion source for ionization and then analyzed by time to pro-mass spectrometry.

\section{Database search and bioinformatics analysis}

First, MaxQuant (v1.6.6.0) was used to retrieve the mass spectrometry data, and Gallus_gallus_UniPort (29475 proteins) database was used. Some parameters in the database were modified and set, including the enzyme restriction method Trypsin/P, to effectively eliminate the influence of contaminated proteins on the identification results and reduce the false positive rate (FDR). The number of missing bits was set to 2, while Firstsearch obtained the first order parent ion mass error tolerance, and the Main search was set as $70 \mathrm{ppm}$ and $70 \mathrm{ppm}$, respectively. The mass error tolerance of secondary fragment ions was set as $0.04 \mathrm{da}$. The fixed modification was set for cysteine alkylation, and variable modification was established for methionine oxidation and N-terminal acetylation and deamidation of protein. The FDR for protein identification and PSM identification was set at $1 \%$. The potential connections and differences on their specific functions (GO, KEGG pathways, protein domains, etc.) were analyzed for biological information based on the functional enrichment and clustering specificity of different groups with differentially expressed proteins (or differentially expressed proteins with different differential multiples),

\section{Parallel reaction monitoring (PRM)}

Specific proteins related to breasts and leg muscles were quantified in LC-PRM/MS by PRM. The results obtained from PRM were calibrated using Xcalibur ${ }^{\text {TM }}$ software. The peptides were separated by an ULTRA high-performance liquid system, injected into an NSI ion source for ionization, and then analyzed by Q ExactiveTM Plus mass spectrometry. 


\section{Results}

\section{Mass spectrometry data analysis of proteome in Jingyuan chicken Leg muscle and Breast muscle}

Label-free quantitative proteomics was used to analyze the Jingyuan chicken leg muscle and Breast muscle tissues. $265,391.0$ secondary protein spectrograms were identified by LC-MS/MS mass spectrometry, and 85811.0 effective spectrograms were obtained after data search, with the utilization rate of $32.3 \%$. After spectrum analysis, $15,139.0$ peptides were identified, among which $12,819.0$ specific peptides were identified. After examination and comparison, 1940.0 proteins were identified, among which 1317.0 proteins could be quantified (Figure 1A). The results showed that the sequence length of detected peptides was mainly distributed between 8 and 32 (Figure 1B). The error between the actual and theoretical values of the identified protein-peptide relative molecular weight was concentrated around 0 , less than $20 \mathrm{ppm}$ (Figure 1C). The analysis found that most peptides were less than $200 \mathrm{kDa}$, and these proteins covered $80 \%$ of the identified protein sequences (Figure 1D). The above data indicated that protein identification results were relatively ideal, and the subsequent data analysis could be continued.

\section{Screening and principal component analysis of differential proteins in leg and Breast muscles of Jingyuan chicken}

By using mass spectrometry, in each protein, the signal abundance was analyzed, and the LFQ intensity value of that protein was obtained using a non-standard quantitative calculation method. Then the calculation of the relative quantitative value of each sample was done according to the protein LFQ intensity value between different samples. The calculation principle of differential protein expression is to screen for differential proteins with a $p$-value of $<0.05$. When the differential protein expression changes by more than 2 , it indicates that the differential protein is upregulated during life-long activities. When the differential protein expression varies less than $1 / 2$, it shows that protein is downregulated during life activities. According to the principle of protein differential expression screening, a total of 190 differentially expressed proteins were found in the muscles of the leg and breasts. Compared to the breast muscles, 121 proteins in the leg muscles were upregulated, and 69 types of proteins were downregulated (Figure 2A, B ). The principal component analysis was carried out on 190 differential proteins identified in Jingyuan chicken leg muscle and breast muscle, as shown in Figure $2 \mathrm{C}$. The variance contribution rate of the first principal component PC1 was $53.8 \%$, the variance contribution rate of the second principal component PC2 was $17.4 \%$. The contribution rate of PC1 and PC2 reached $71.2 \%$, reflecting most of the information on the change of differential proteins, and the proteome of the two parts was significantly different.

\section{Functional classification and GO enrichment analysis of differentially expressed proteins in Jingyuan chicken leg muscle and breast muscle}

To further study the biological functions performed by differentially expressed proteins in Jingyuan chicken leg and breast muscles, we performed Gene Ontology (GO) function annotation and enrichment analysis of 190 differential proteins. Mainly through the biological process (BP), cellular component (CC), 
and molecular function (MF) are three aspects of the differentially expressed proteins that have been elaborated. Figure 3 shows that differential proteins are mainly involved in cellular processes (116 proteins, $22 \%$ ), single-organism process (112 proteins, $21 \%$ ), metabolic processes (93 proteins, 18\%) ), biological regulation (47 proteins, accounting for $9 \%$ ), cellular component organization or biogenesis (33 proteins, accounting for $6 \%$ ) and other biological processes. In CCs, differential proteins are mainly involved in the cell (114 differential proteins, accounting for $30 \%$ ), organelle (87 proteins, accounting for $23 \%$ ), macromolecular complex (51 proteins, accounting for $14 \%$ ), membrane (49 proteins, accounting for $13 \%$ ), extractor region (38 proteins, accounting for $10 \%$ ) and other cell components. In MFs, differential proteins mainly participate in binding (123 proteins, accounting for $45 \%)$, catalytical activity (104 proteins, accounting for $38 \%$ ), transportation activity (16 proteins, accounting for $6 \%$ ), structural molecular activity (11 proteins, accounting for $4 \%$ ) and other molecular functions.

Further, the subcellular localization of 190 differentially expressed proteins found in leg muscle and breast muscle of Jingyuan chicken. The differential proteins were mainly located in the cytoplasm (92 proteins, $48.42 \%$ ), mitochondria ( 28 proteins, $14.74 \%)$, nucleus (24 proteins, $12.63 \%$ ), extracellular (21 protein, $11.05 \%$ ), cytoplasm nucleus (12 proteins, $6.32 \%$ ), plasma membrane ( 9 proteins, $4.74 \%$ ), other (4 proteins, $2.11 \%$ ) organelles, as shown in Fig. 4. For systematically analyzing the differential protein functions identified in Jingyuan chicken leg and breast muscles, COG (Cluster of Orthologous Groups of proteins) was used to perform gene function annotation and genome evolution analysis on 190 differential proteins. Figure 5 shows the results obtained from the study. The Histogram shows participation in energy production and conversion (15 proteins), carbohydrate transportation and metabolism (15 proteins), lipid transportation and metabolism (13 proteins), cytoskeleton (12 proteins), general function prediction (9 proteins), translation, ribosomal structure and biogenesis (5 proteins), intracellular trafficking, secretion and vesicular transport (5 proteins), etc. The biological processes with the highest frequency of proteins are primarily energy production and conversion (15 proteins), carbohydrate transport, and metabolism (15 proteins), lipid transport, and metabolism (13 proteins). The biological processes with the lowest frequency of occurrence mainly include post-translational modification, protein turnover, chaperones, coenzyme transport and metabolism, Inorganic ion transport and metabolism, secondary metabolites biosynthesis, transport, and catalyst, etc. However, in the annotation of gene function and genome evolution analysis, 90 different protein functions have not been determined.

\section{KEGG pathway analysis of differentially expressed protein in Jingyuan chicken leg muscle and breast muscle}

Research shows that many types of proteins in the body perform their biological functions through interaction, and a single protein cannot independently perform its biological functions as the interaction of these proteins is connected by signal pathways. Therefore, the analysis of different pathways of proteins can help us to understand the biological functions more systematically and comprehensively. The regulatory mechanism of protein-protein interaction involves a change of character or occurrence of diseases. The KEGG analysis method may well classify proteins, identify the metabolic or signaling 
pathways in which these proteins may participate, and obtain information about the proteins involved in a series of biological processes from the cell surface to the nucleus, which ultimately helps us understand the entire organism. KEGG pathway enrichment analysis of 190 proteins differentially expressed in Jingyuan chicken leg muscles and breast muscles showed that 190 differential proteins involved a total of 286 signaling pathways, of which these differential proteins involved more than 20 significant pathways: gga01100 Metabolic pathways, gga00500 Starch and sucrose metabolism, Figure 6 depicts gga00010 Glycolysis/Gluconeogenesis, gga00071 Fatty acid degradation, gga01212 Fatty acid metabolism, gga01200 Carbon metabolism, gga00280 Valine, leucine and isoleucine degradation, gga03320 PPAR signaling pathway, etc. Thus, proteins with a significant difference between Jingyuan chicken leg muscle and breast muscle are mainly enriched by gga000500 starch and sucrose metabolism, gga00071 Fatty acid degradation, gga01212 fatty acid metabolism, gga03320 PPAR signaling pathway, etc.

\section{Parallel reaction monitoring (PRM) validation of the differential expression protein between leg muscle and breast muscle of Jingyuan chicken}

According to the information on the differential protein peptides in combination with $\mathrm{GO}$ analysis and KEGG pathway analysis, 23 target proteins were selected for PRM quantification. Due to the characteristics of some proteins and the abundance of their expression, only 21 of them have been quantified. It was found that 10 of the 21 quantified proteins were related to fat deposition, namely E1BTT4, E1C0Q5 (ACAT1), F1NC38 (ACADL), Q5F420 (ACSL1), A0A1D5P3S9 (ECI1), Q5ZL56 (ACADS), F1NUQ3 (FABP3), A0A1D5PL36 (ACAA2), F1NR44 (ECHS1), Q5ZIR7 (FABP5) and other ten proteins. These proteins were enriched in "gga00071Fattyaciddegradation, gga01212Fattyacidmetabolis, gga03320PPARsignalingpathway" network pathways that regulate fat deposition, as shown in Table1. The activity of related proteins that regulate fat deposition in Jingyuan chicken leg muscles was found to be significantly more potent than that of breast muscles, further explaining the critical reason why the IMF content of leg muscles was higher than that of breast muscles.

\section{Network Interaction Analysis of IMF-specific Deposited Key Proteins in Jingyuan Chicken Leg and breast Muscles}

Protein is the primary executor of life activities, and the interaction between proteins plays a vital role in regulating life activities. Both STRING and Cytoscape software were used to analyze the network interactions of IMF specific deposition proteins that regulated the leg and breast muscles of Jingyuan chicken to obtain a protein-protein interaction network map. Figure 7 depicts that there are eight proteins involved in the protein-protein interaction network, and these eight proteins form 19 network interaction paths, indicating that IMF deposition is a very complex and ordered biological process.

\section{Discussion}

Meat quality is a comprehensive economic character, including flavor, texture, nutrition, safety, and so on. Many factors are affecting the quality of domestic chicken, including muscle $\mathrm{pH}$, temperature, the water 
activity of the reaction system, the interaction of flavor, and the composition of the meat matrix. The factors of production management mainly include breed, age, sex, dietary factors, feeding environment, feeding system, slaughter process, conditions for carcass storage, cooking methods, etc. which have different degrees of influence on the taste of poultry meat (Wang Shubo 2004; Guang-min zhang, 2006; Lu et al. 2007). IMF has a specific relationship with muscle tenderness, juiciness, flavor, and shear strength that can significantly boost the quality of meat (Fernandez et al. 1999; Jeremiah et al. 2003; Hambrecht et al. 2005; Qiankun et al. 2010; Zhang et al. 2018; Wang Qin 2019). It is mainly reflected in two aspects: on the one hand, fat content in muscle is positively correlated with muscle tenderness (Okeudo N \& Moss B 2005; Chartrin et al. 2006) because of the adipose tissue and connective tissue in the muscle are in a state of the intersection. This morphological structure may loosen the connective tissue and decrease the physical strength of the connective tissue so that the muscle fiber bundle can be easily separated, and the muscle tenderness can be improved. At the same time, oxidation has the effect of dissolving muscle fiber bundles, and can also enhance muscle tenderness and juiciness to a certain extent (Blanchard et al. 2000; Fortin et al. 2005). In contrast, intramuscular fat is rich in phospholipids (approximately $0.5 \%-1 \%$ of fresh muscle weight). Phospholipids contain a large amount of polyunsaturated fatty acids, especially long-chain polyunsaturated fatty acids. Thus, it can generate aroma substances through the Maslard reaction (JO \& AM 1979; Ghasemi $\mathrm{H}$ et al. 2016), thereby increasing the flavor of the meat. By studying the IMF content in the breast muscles of the selected five generations of yellow feather broilers, it was found that increasing the IMF content in the muscle could increase the muscle tenderness and thus increase the sexual maturity time of the chicken (Zhao G et al. 2007). It can be proved that the deposition of IMF content in muscle plays a key role in improving the flavor and quality of muscle.

Genetic and nutritional factors regulate IMF deposition, which has a medium genetic factor $(\mathrm{h} 2=0.5)$ in the present studies. Differences in animal species, sex, age, tissue location, etc., cause discrepancies in IMF deposition (Li Huifang and Chen Kuanwei 2004; Jiang Ruirui 2010). Studies have found that the IMF content of leg muscles is significantly higher than that of breast muscles, which is 3-4 times that of breast muscles (Li Wenjuan 2008). Based on the comparative analysis of IMF content in different days and parts of broilers, it is clear that IMF content increased with the increase of days in 28-90 days (Chen Jilan et al. 2005). These studies showed variations in time and tissue of fat deposition in different sections of tissue. The mechanism may be related not only to lipid synthesis and degradation under the influence of hormones and enzymes in different parts of the adipose tissue but also to a certain degree related to the variation in the state of development of microvascular tissue (Leclercq et al. 1984). The deposition of fat in the body has a precise temporal and spatial sequence (Griffin et al. 1987). The measurement results show that the sequence of fat deposition sites in broilers is abdominal organs, subcutaneous tissues, muscle bundles, and finally, muscle fibers (Wang et al. 2009; Wu et al. 2016). The high-intensity breeding selection causes the growth speed of the broiler rapidly, almost reaching its physiological limit, leading to a major reduction in the quality of the meat. Rapid growth causes excessive fat accumulation in the abdomen and internal organs, resulting in a certain amount of waste of resources 
and environmental pollution. Therefore, improving the IMF and the meat quality while broilers are rapidly growing is a hot topic in the current research direction of broiler breeding.

Based on the previous results obtained in this project, Jingyuan chicken, a high-quality local chicken species in China, was selected as the objective of the present research. By studying lab free proteomics on the leg and breast muscles of Jingyuan chicken, it was found that there were 190 differentially expressed proteins between them, of which 121 proteins were upregulated, and 69 proteins were downregulated. According to the $\mathrm{GO}$ analysis of 190 differential proteins, 93 proteins participated in the biological process of metabolic process, while 47 proteins participated in the biological regulation. These biological processes and activities play an essential role in regulating fat deposition, which may be one of the reasons why the IMF content of leg muscle is higher than that of breast muscle. Moreover, the subcellular localization of 190 differentially expressed proteins showed that they were mainly located in cytoplast (92 proteins, $48.42 \%$ ), mitochondria ( 28 proteins, $14.74 \%$ ), nucleus ( 24 proteins, $12.63 \%$ ) and other subcells. Among them, mitochondria are the central place for conducting aerobic respiration in cells where $95 \%$ of the energy required for cell life activities comes from mitochondria, which contributes to the metabolism of substances and the deposition of fat. The analysis of 190 differential proteins by COG showed that only 100 of them could be identified. Among them, 43 were related to the synthesis and degradation of fat. They participated in energy production and conversion (15 proteins), carbohydrate transport and metabolism (15 proteins), lipid transport and metabolism (13 proteins), and other biological functions. Therefore, it should be noted that the difference in fat deposition between leg muscle and breast muscle is primarily due to the difference in the biological functions of essential proteins controlling the synthesis and degradation of fat.

Using KEGG to analyze the pathways of differentially expressed proteins, it was found that the proteins with significant differences were mainly enriched by pathways related to sugar metabolism and fat deposition such as ga00500 starch and sucrose metabolism, gga00071 fatty acid degradation, gga01212 fatty acid metabolism, and gga03320 PPAR signaling pathway. Hence, the differential protein may regulate the differential deposition of IMF in leg muscles and breast muscles through glucose metabolism, fat synthesis, and degradation. The results of the present study further interpreted the biological characteristics of IMF specific deposition between Jingyuan chicken leg muscles and breast muscles.

PRM was used to quantify 190 proteins with differential expression, of which 23 of them were selected for PRM quantification. Due to the characteristics of certain proteins and the frequency of their expression, only 21 of them have been quantified. Further analysis found that among the 21 proteins quantified, ten proteins that regulate fat deposition have major variations in expression between leg muscles and breast muscles. For example, Acetyl CoA Acyltransferase 2 (AcetylCoAacyltransferase 2, ACAA2) is a key enzyme for fatty acid oxidation, widely distributed in mammalian and bird tissues and cytoplasm, and plays a vital role in the metabolism of fatty acids (Sodhi et al. 2014), and regulates intermuscular fat content (Miltiadou et al. 2017). Hence, this gene is considered a candidate for detecting intermuscular fat content (Miltiadou et al. 2017). In additionखanother study showed that the expression of 
the ACAA2 gene in chicken leg muscles was significantly higher than that of breast muscles (Liu Chen 2009).

Long-chain acyl-CoA dehydrogenase (Acyl-CoAdehydroge-nase, long-chain ACADL) is a coenzyme of acetylase in the body that plays an important role in the metabolism of carbohydrates, proteins, and lipids, particularly in long-chain $\beta$-oxidation fatty acids, and its regulation mechanism mainly includes regulations of expression and activity, has a very close relationship (Luan Yihong et al. 2003; Li Peng 2012; Lu Zhaoning et al. 2013). Studies have shown that lack of ACADL can cause mitochondrial dysfunction and liver steatosis or liver insulin resistance (Ji \& Friedman 2007; Zang Shasha et al. 2014), in particular, ACADL plays an important role in the liver metabolism of chickens ( Yue Ying 2013). Shortchain acyl-CoA dehydrogenase (Acyl-CoA dehydrogenase short-chain ACADS) is a non-acyl dehydrogenase that is primarily responsible for $\beta$-oxidation of C4-C6 short-chain fatty acids and is also the rate-limiting enzyme in fatty acid degradation (Kruger et al. al. 2012). Previous studies have shown that ACADS deficiency can lead to the disorder symptoms of fat conversion to energy loss, such as drowsiness, hypoglycemia, hypotension (Chen \& Su 2015), and inborn energy metabolism errors (Vockley 2008; Peng et al. 2013). Based on these data, we believe that ACADS plays a key role in maintaining energy balance in the fatty acid degradation pathway.

ACSL1 is involved in the synthesis of triglycerides by fatty acyl-CoA, promotes FA depositions (Pan Zhixiong et al. 2010), activates FA, and enters the $\beta$-oxidation pathway (Ellis et al. 2010). According to reports, the ACSL1 gene affects the fatty acid composition by regulating the overall fat content of the skeletal muscle (Young et al. 2018). Acetyl coenzyme A acetyltransferase 1 (ACAT1) is a type of mitochondrial enzyme that transforms two acetyl-coenzyme $A$ molecules into acetyl coenzyme $A$ and coenzyme $A$ in the process of ketogenesis (Haapalainen et al. 2005). Earlier, ACAT1 and SIRT3 were used as upstream acetyltransferase and deacetylase for PDHA1 and PDP1, respectively (Fan et al. 2014). Moreover, long-chain fatty acyl-CoA synthetase (ACSL) is a crucial enzyme for fatty acid (FA) activation and catalysis, which can convert long-chain fatty acids into fatty acyl-CoA. It has been found that the ACSL1 gene encodes a synthetase related to lipid metabolism, mainly expressed in adipose tissue and liver intima (Wang Qianqian et al. 2016).

Enoyl-CoA hydratase short chain 1 (ECHS1) is a key enzyme that catalyzes the second step in the physiologically crucial $\beta$-oxidation pathway of fatty acid metabolism (Janssen et al. 1997; Liu Haifeng et al. 2010). The decrease of the expression of echs 1 will cause the reduction of fatty acid $\beta$ oxidation, so that a large number of fatty acids stored in the liver cannot be oxidized for energy supply, resulting in the accumulation of fat in the liver cells. Besides, ECHS1 is an essential gene involved in cholesterol metabolism (Liu Rong et al. 2008). Earlier reports have found that a lack of ECHS1 expression causes metastatic conjunctival melanoma (Lake et al. 2011), while significant downregulation of ECHS1 expression leads to renal cell carcinoma and hepatocellular carcinoma (Yokoyama et al. 2004; Hwa et al. . 2005). In contrast, $\Delta 3-\Delta 2$-enoyl-CoA isomerase (ECl1) is a coenzyme necessary for the metabolism of double bonds in the process of $\beta$ oxidation (Kilponen \& Hiltunen 1993; Yun-feng et al. 2017) and plays an important role during the metabolism of unsaturated fatty acids (Gurvitz et al. 1999; Hiltunen \& Qin 2000; 
Fumiyo et al. 2010). Studies have shown that the expression of the ECl1 gene in Chinese pig back has the longest muscle is significantly higher than that of other foreign lean meat pigs, indicating that it is likely to be the primary gene-regulating pig fat metabolism (Lu Yunfeng et al. 2015).

Heart fatty acid-binding protein (FABP3 or H-FABP) is a member of the fatty acid-binding protein family, which expressed in the myocardium and skeletal muscle (Veerkamp \& Maatman 1995). It is involved in maintaining the energy balance of the heart and other body tissues, regulating muscle fat content, and the development of fatty tissues (Binas et al. 1999; Shearer et al. 2005; Li et al. 2007). The overexpression of the FABP3 gene in 3T3-L1 preadipocytes can promote fat synthesis (Yi et al. 2014). The results showed that the level of FABP3 gene mutation and mRNA expression in livestock and poultry had a significant effect on IMF ( $\mathrm{Li}$ et al. 2010; Cho et al. 2011), which affected muscle tenderness. For example, the mutation of the FABP3 gene has an essential impact on the IMF content of pigs (Wang et al. 2015) and goats (Sweeney et al. 2015). Therefore, researchers studied the FABP3 gene as a candidate gene for regulating the IMF and tenderness of domestic animals. The effect of FABP3 on the IMF and the meat quality is mainly to maintain the concentration gradient of intracellular and extracellular fatty acids by combining fatty acids, to promote the entry of fatty acids into cells to improve IMF content (Atay et al. 2019; Shang et al. 2019) and muscle tenderness (Li Wufeng et al. 2004; Zhou Guoli et al. 2005). Fatty acid-binding protein 5 (FABP5), also known as epidermal fatty acid-binding protein (E-FABP), belongs to the family of fatty acid-binding proteins (FABPs), is an intracellular lipid carrier (Yu Mengmeng. 2017). Based on studies, FABPS has a high affinity for stearic and linoleic acids and binds explicitly with fatty acids (FA), and regulate the lipid metabolism by controlling intracellular fatty acid transport (Dallaglio et al. 2013). Besides, FABP5 also plays a vital role in regulating cell proliferation, metabolism, and immune response (Yu et al. 2016). Researchers have found that FABPS can activate the PPARR pathway by combining long-chain fatty acids (Yu et al. 2012). Furthermore, several studies have shown that FABP5 is significantly related to fat traits and production performance of livestock and poultry (Estellé et al. 2006; Yu Jiahui 2007; Ojeda et al. 2008; Zhang Qingqiu et al. 2012).

By analyzing ten differential proteins, we found that these differential proteins are significantly related to fat deposition, and demonstrate that regulating fat deposition is not a simple biological process that a single protein or gene can complete. So far, no reports are available regarding the mechanism of specific deposition of IMF in different parts of Jingyuan chicken. We used a mathematical program (STRING and Cytoscape software) to perform the network interactions on ten screened proteins and mapped between them. We found that only eight proteins among ten have participated in network interaction, constituting a total of 19 relatively complex network pathways.

\section{Conclusion}

To conclude, we used label-free proteomics and PRM methods to carry out basic research on the leg and breast muscles of Jingyuan chicken. This study not only explains the molecular mechanism of IMF specific deposition between the leg muscles and the breast muscles of Jingyuan chicken but also analyzes the network interaction of essential functional proteins regulating IMF deposition. In this study, 
we conclude that the key proteins regulate the synthesis and degradation of fat in different parts of the body through biological processes. The value and significance of these results are mainly reflected in that they not only provide molecular materials for the study of increasing IMF content of broilers but also establish an ideal animal model for the future research of meat quality. Hence, the present research has typical biological significance for the molecular breeding of high-quality local broilers.

\section{Abbreviations}

IMF:Intramuscular fat;

PRM:parallel reaction monitoring method;

GO:Gene Ontology;

BP:Biological process;

CC:Cellular component ;

MF: Molecular function;

COG:Cluster of Orthologous Groups of proteins

\section{Declarations}

\section{Acknowledgments}

The authors thank all animal breeders and farmers who provided the raw material for the experiments. The authors are also grateful to all the members of the research group for their contribution.

\section{Funding}

This study was funded by the National Natural Science Foundation of China "Identification and Regulation Mechanism of Key MiRNAs and IncRNAs in Jingyuan Chicken Muscle Specific Deposits (31860621)". These funds are mainly used for experimental design, data processing and analysis, animal feeding and collection, and the publication of articles.All funds are used under the supervision and approval of the National Foundation of China and Ningxia University.

\section{Availability of data and materials}

Not applicable

\section{Authors' contributions}

ZWH and JZ designed the study, performed the statistical analyses, and drafted the manuscript. ZYC,XFF andGSX wrote the manuscript and analyzed the data. YLG,TM,CYY and XFY revised the manuscripts. All 
authors were involved in interpreting and discussing the results, and have read and approved the final manuscript.

\section{Ethics approval}

The experiment was performed according to the animal care and following the guidelines of the Animal Care Committee at Ningxia University.Postoperative animals according to animal harmless treatment methods and principles, scientific treatment.

\section{Consent for publication}

Consent for publication

\section{Competing interests}

The authors declare that they have no competing interests.

\section{Author address}

Animal Genetics, Breeding and Reproduction of Ningxia University Agricultural College, P.O.Box 750, 021 Yinchuan $\bowtie$ Neijiang Vocational and Technical College, Dongxing District, Neijiang City, Sichuan Province, P.O.Box 641,000 .

\section{References}

1. Atay E, Guzel M, Amanvermez R, Demir MT, Erenler AK, Ozgen E \& Yucel Mø2019®: Role of Gal-3 and H-FABP in the early diagnosis of acute coronary syndrome. Bratisl Lek Listy 120: 124-130.

2. Binas B, Danneberg H, Mcwhir J, Mullins L \& Clark AJ囚1999『: Requirement for the heart-type fatty acid binding protein in cardiac fatty acid utilization. The FASEB Journal 13 .

3. Blanchard PJ, Willis MB, Warkup CC \& Ellis M\&2000冈: The influence of carcass backfat and intramuscular fat level on pork eating quality. J Sci Food Agric 80 .

4. Chartrin P, Méteau K, Juin H, Bernadet M D, Guy G, Larzul C, Rémignon H, Mourot J, Duclos M J \& Baéza $E \llbracket 2006 \rrbracket:$ Effects of intramuscular fat levels on sensory characteristics of duck breast meat. Poult Sci 85 .

5. Chen Y \& Su Zヌ2015囚: Reveal genes functionally associated with ACADS by a network study. Gene 569 .

6. Cho KH, Kim MJ, Jeon GJ \& Chung HY『2011囚: Association of genetic variants for FABP3 gene with back fat thickness and intramuscular fat content in pig. Mol Biol Rep 38 .

7. Cui H, Zheng M, Zhao G, Liu R \& Wen J囚2018®: Identification of differentially expressed genes and pathways for intramuscular fat metabolism between breast and thigh tissues of chickens. BMC Genomics 19: 55. 
8. Dallaglio K, Marconi A, Truzzi F, Lotti R, Palazzo E, Petrachi T, Saltari A, Coppini M \& Pincelli C $\varangle 2013 \rrbracket$ : E-FABP induces differentiation in normal human keratinocytes and modulates the differentiation process in psoriatic keratinocytes in vitro. Exp Dermatol 22 .

9. Ellis JM, Li LO, Wu PC, Koves TR, Ilkayeva O, Stevens RD, Watkins SM, Muoio DM \& Coleman RA $\varangle 2010 \rrbracket$ : Adipose acyl-CoA synthetase- 1 directs fatty acids toward beta-oxidation and is required for cold thermogenesis. Cell Metab 12: 53-64.

10. Estellé J, Pérez-Enciso M, Mercadé A, Varona L, Alves E, Sánchez A \& Folch JMヌ2006®:

Characterization of the porcine FABP5 gene and its association with the FAT1 QTL in an Iberian by Landrace cross. Anim Genet 37 .

11. Fan J, Shan C, Kang H, Elf S, Xie J, Tucker M, Gu T, Aguiar M, Lonning S, Chen H, Mohammadi M, Britton LP, Garcia BA, Alečković M, Kang Y, Kaluz S, Devi N, Van Meir EG, Hitosugi T, Seo JH \& Sagar.. \2014邓: Tyr Phosphorylation of PDP1 Toggles Recruitment between ACAT1 and SIRT3 to Regulate the Pyruvate Dehydrogenase Complex. Mol Cell 53 .

12. Fernandez X, Monin G, Talmant A, Mourot J \& Lebret B囚1999囚: Influence of intramuscular fat content on the quality of pig meat -1 . Composition of the lipid fraction and sensory characteristics of $m$. longissimus lumborum. Pubmed 53 .

13. Fortin A, Robertson W M \& Tong A K W囚2005区: The eating quality of Canadian pork and its relationship with intramuscular fat. Meat Sci 69 .

14. Fumiyo, Kasuya, Ryota, Nishizawa, Teiichi, Masuyama, Maya \& Kazumið2010®: Evaluation of a screening method by liquid chromatography-tandem mass spectrometry for estimating effect of drugs on the activation and $<\mathrm{em}>\beta</ \mathrm{em}>-$ oxidation of fatty acids in mitochondria. $\mathrm{J}$ Pharm Pharmacol.

15. Ghasemi H A, Shivazad M, Mirzapour Rezaei S S \& Karimi Torshizi M A囚2016囚: Effect of synbiotic supplementation and dietary fat sources on broiler performance, serum lipids, muscle fatty acid profile and meat quality. Br Poult Sci 57 .

16. Griffin HD, Butterwith SC \& Goddard C $₫ 1987 \rrbracket$ : Contribution of lipoprotein lipase to differences in fatness between broiler and layer-strain chickens. Br Poult Sci 28: 197-206.

17. Guan L, Hu X, Liu L, Xing Y, Zhou Z, Liang X, Yang Q, Jin S, Bao J, Gao H, Du M, Li J \& Zhang L $\triangle 2017 \rrbracket$ : bta-miR-23a involves in adipogenesis of progenitor cells derived from fetal bovine skeletal muscle. Sci Rep 7: 43716.

18. Gurvitz A, Wabnegger L, Yagi Al, Binder M, Hartig A, Ruis H, Hamilton B, Dawes IW, Hiltunen JK \& Rottensteiner H囚1999『: Function of human mitochondrial 2,4-dienoyl-CoA reductase and rat monofunctional Delta3-Delta2-enoyl-CoA isomerase in beta-oxidation of unsaturated fatty acids. Biochem J 344 Pt 3: 903-914.

19. Haapalainen AM, Meriläinen G \& Wierenga RK囚2005『: The thiolase superfamily: condensing enzymes with diverse reaction specificities. Trends Biochem Sci 31 .

20. Hambrecht E, Eissen JJ, Newman DJ, Smits CH, Verstegen MW \& den Hartog LA囚2005囚: Preslaughter handling effects on pork quality and glycolytic potential in two muscles differing in fiber type 
composition. J Anim Sci 83: 900-907.

21. Hiltunen JK \& Qin YMヌ2000®: $\beta$-Oxidation - Strategies for the metabolism of a wide variety of acylCoA esters. Biochim Biophys Acta 1484: 117-128.

22. Hwa JS, Park HJ, Jung JH, Kam SC, Park HC, Kim CW, Kang KR, Hyun JS \& Chung KH囚2005囚: Identification of proteins differentially expressed in the conventional renal cell carcinoma by proteomic analysis. J Korean Med Sci 20: 450-455.

23. O. I \& A. M. P囚1979®: ROLE OF PHOSPHOLIPIDS AND TRIGLYCERIDES IN WARMED-OVER FLAVOR DEVELOPMENT IN MEAT MODEL SYSTEMS. John Wiley \& Sons, Ltd (10.1111) 44 .

24. Janssen U, Davis EM, Le Beau MM \& Stoffel W囚1997囚: Human mitochondrial enoyl-CoA hydratase gene (ECHS1): structural organization and assignment to chromosome 10q26.2-q26.3. Genomics 40: 470-475.

25. Jeremiah LE, Dugan ME, Aalhus JL \& Gibson LL『2003囚: Assessment of the relationship between chemical components and palatability of major beef muscles and muscle groups. Meat Sci 65 : 1013-1019.

26. Ji H \& Friedman MIه2007囚: Reduced capacity for fatty acid oxidation in rats with inherited susceptibility to diet-induced obesity. Metabolism 56: 1124-1130.

27. Jiang M, Fan WL, Xing SY, Wang J, Li P, Liu RR, Li QH, Zheng MQ, Cui HX, Wen J \& Zhao GP区2017区: Effects of balanced selection for intramuscular fat and abdominal fat percentage and estimates of genetic parameters. Poult Sci 96: 282-287.

28. Kilponen JM \& Hiltunen JK囚1993囚: $\beta$-Oxidation of unsaturated fatty acids in humans Isoforms of $\Delta 3$, $\triangle 2$-enoyl-CoA isomerase .

29. Kruger C, Kumar KG, Mynatt RL, Volaufova J \& Richards BK囚2012区: Brain transcriptional responses to high-fat diet in Acads-deficient mice reveal energy sensing pathways. PLoS One 7: e41709.

30. Lake SL, Jmor F, Dopierala J, Taktak AFG, Coupland SE \& Damato BE 2011 : : Multiplex LigationDependent Probe Amplification of Conjunctival Melanoma Reveals Common BRAF V600E Gene Mutation and Gene Copy Number Changes. Invest Ophthalmol Vis Sci 52 .

31. Laura B, Rosa C \& Carlos A囚2017囚: Microbial loads and antibiotic resistance patterns of Staphylococcus aureus in different types of raw poultry-based meat preparations. Poult Sci 96 .

32. Leclercq B, Hermier D \& Salichon M R⿴1984『: Effects of age and diet on plasma lipid and glucose concentrations in genetically lean or fat chickens. Reproduction, nutrition, developpement 24 .

33. Li A, Zhang Y, Zhao Z, Wang M \& Zan L区2016冈: Molecular Characterization and Transcriptional Regulation Analysis of the Bovine PDHB Gene. PLoS One 11: e0157445.

34. Li B, Zerby HN \& Lee K囚2007囚: Heart fatty acid binding protein is upregulated during porcine adipocyte development. J Anim Sci 85: 1651-1659.

35. Li Q, Zuo LL, Lin YQ, Xu YO, Zhu JJ, Liao HH, Lin S, Xiong XR \& Wang Yヌ2016『: Cloning and Expression of SFRP5 in Tibetan Chicken and its Relationship with IMF Deposition. Anim Biotechnol 27: 231-237. 
36. Li X, Kim S, Choi J, Lee Y, Lee C, Choi B, Kim T, Choi Y, Kim J \& Kim K囚2010囚: Investigation of porcine FABP3 and LEPR gene polymorphisms and mRNA expression for variation in intramuscular fat content. Mol Biol Rep 37 .

37. Liu L, Cui H, Fu R, Zheng M, Liu R, Zhao G \& Wen J囚2017区: The regulation of IMF deposition in breastis major of fast- and slow- growing chickens at hatching. J Anim Sci Biotechnol 8: 77.

38. Liu R, Wang H, Liu J, Wang J, Zheng M, Tan X, Xing S, Cui H, Li Q, Zhao G \& Wen J囚2017囚: Uncovering the embryonic development-related proteome and metabolome signatures in breast muscle and intramuscular fat of fast-and slow-growing chickens. BMC Genomics 18: 816.

39. Liu R, Zheng M, Wang J, Cui H, Li Q, Liu J, Zhao G \& Wen J『2019邓: Effects of genomic selection for intramuscular fat content in breast muscle in Chinese local chickens. Anim Genet 50: 87-91.

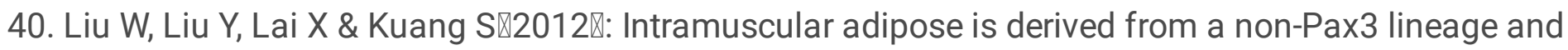
required for efficient regeneration of skeletal muscles. Dev Biol 361: 27-38.

41. Lu Q, Wen J \& Zhang $H \otimes 2007 \rrbracket:$ Effect of chronic heat exposure on fat deposition and meat quality in two genetic types of chicken. Poult Sci 86: 1059-1064.

42. Ma JS, Chang WH, Liu GH, Zhang S, Zheng AJ, Li Y, Xie Q, Liu ZY \& Cai HY『2015区: Effects of flavones of sea buckthorn fruits on growth performance, carcass quality, fat deposition and lipometabolism for broilers. Poult Sci 94: 2641-2649.

43. Miltiadou D, Hager-Theodorides AL, Symeou S, Constantinou C, Psifidi A, Banos G \& Tzamaloukas 0 ه2017邓: Variants in the 3' untranslated region of the ovine acetyl-coenzyme A acyltransferase 2 gene are associated with dairy traits and exhibit differential allelic expression. J Dairy Sci 100: 6285-6297.

44. Muazzez C, Yılmaz Ş, Figen K, Hakan BÖ \& Selim M『2016邓: Comparison of Carcass Characteristics, Meat Quality, and Blood Parameters of Slow and Fast Grown Female Broiler Chickens Raised in Organic or Conventional Production System. Asian-Australas J Anim Sci 29.

45. Ojeda A, Estellé J, Folch JM \& Pérez-Enciso Mヌ2008®: Nucleotide variability and linkage disequilibrium patterns at the porcine FABP5 gene. Anim Genet 39: 468-473.

46. Okeudo N J \& Moss B W $\otimes 2005 \rrbracket:$ Interrelationships amongst carcass and meat quality characteristics of sheep. Meat Sci 69 .

47. Peng Y, Xiang H, Chen C, Zheng R, Chai J, Peng J \& Jiang S $₫ 2013 \rrbracket:$ MiR-224 impairs adipocyte early differentiation and regulates fatty acid metabolism. The international journal of biochemistry and cell biology 45: 1585-1593.

48. Qiankun Z, Hong-Gu L, Jung-A H, Bae KE, Kee KS, Jinlong Y, Myunggi B, Yannan S, Sang-Hoon K, Kang-Seok S \& Yun-Jaie C $₫ 2010 \rrbracket$ : Differentially expressed proteins during fat accumulation in bovine skeletal muscle. Meat Sci 86 .

49. Shang P, Zhang B, Zhang J, Duan M, Wu L, Gong X, Tang K, Zhang H \& Chamba Y®2019®: Expression and single-nucleotide polymorphisms of the H-FABP gene in pigs. Gene 710: 156-160.

50. Shearer J, Fueger PT, Rottman JN, Bracy DP, Binas B \& Wasserman DH $2005 \rrbracket$ : Heart-type fatty acidbinding protein reciprocally regulates glucose and fatty acid utilization during exercise. Am J Physiol Endocrinol Metab 288: E292-297. 
51. Sodhi SS, Ghosh M, Song KD, Sharma N, Kim JH, Kim NE, Lee SJ, Kang CW, Oh SJ \& Jeong DK \2014『: An approach to identify SNPs in the gene encoding acetyl-CoA acetyltransferase-2 (ACAT-2) and their proposed role in metabolic processes in pig. PLoS One 9: e102432.

52. Sweeney T, O'Halloran AM, Hamill RM, Davey GC, Gil M, Southwood OI \& Ryan MT囚2015囚: Novel variation in the FABP3 promoter and its association with fatness traits in pigs. Meat Sci 100 .

53. Veerkamp JH \& Maatman RGHJ囚1995囚: Cytoplasmic fatty acid-binding proteins: Their structure and genes. Prog Lipid Res 34 .

54. Vockley $\mathrm{J} \otimes 2008 \rrbracket$ : Metabolism as a complex genetic trait, a systems biology approach: Implications for inborn errors of metabolism and clinical diseases. J Inherit Metab Dis 31 .

55. Wang B, Fu X, Liang X, Deavila JM, Wang Z, Zhao L, Tian Q, Zhao J, Gomez NA, Trombetta SC, Zhu

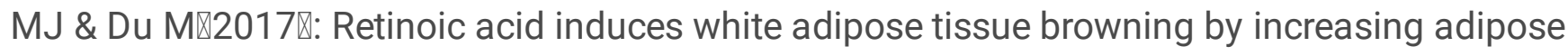
vascularity and inducing beige adipogenesis of PDGFRa+ adipose progenitors. Cell Discov 3: 17036.

56. Wang D, Wang N, Li N \& Li H囚2009『: Identification of differentially expressed proteins in adipose tissue of divergently selected broilers. Poult Sci 88 .

57. Wang L, Li L, Jiang J, Wang Y, Zhong T, Chen Y, Wang Y \& Zhang H囚2015区: Molecular characterization and different expression patterns of the FABP gene family during goat skeletal muscle development. Mol Biol Rep 42 .

58. Wang Y, Chen H, Han D, Chen Y, Muhatai G, Kurban T, Xing J \& He J®2017 \: Correlation of the A-FABP Gene Polymorphism and mRNA Expression with Intramuscular Fat Content in Three-Yellow Chicken and Hetian-Black Chicken. Anim Biotechnol 28: 37-43.

59. Wang Y, Hui X, Wang H, Kurban T, Hang C, Chen Y, Xing J \& Wang J『2016区: Association of H-FABP gene polymorphisms with intramuscular fat content in Three-yellow chickens and Hetian-black chickens. J Anim Sci Biotechnol 7: 9.

60. Wu C, Wu Y, Liu C, Wang Y, Na W, Wang N \& Li H囚2016冈: Comparative proteome analysis of abdominal adipose tissues between fat and lean broilers. Proteome Sci 14 .

61. Yi B, Wang J, Wang S, Yuan D, Sun J, Li Z, Mao Y, Hou Q \& Liu W囚2014ম: Overexpression of Banna mini-pig inbred line fatty acid binding protein 3 promotes adipogenesis in 3T3-L1 preadipocytes. Cell Biol Int 38: 918-923.

62. Yokoyama Y, Kuramitsu Y, Takashima M, lizuka N, Toda T, Terai S, Sakaida I, Oka M, Nakamura K \& Okita K囚2004『: Proteomic profiling of proteins decreased in hepatocellular carcinoma from patients infected with hepatitis C virus. Proteomics 4: 2111-2116.

63. Young PA, Senkal CE, Suchanek AL, Grevengoed TJ, Lin DD, Zhao L, Crunk AE, Klett EL, Füllekrug J, Obeid LM \& Coleman RA囚2018囚: Long-chain acyl-CoA synthetase 1 interacts with key proteins that activate and direct fatty acids into niche hepatic pathways. J Biol Chem 293: 16724-16740.

64. Yu C, Liang X, Lipsky S, Karaaslan C, Kozakewich H, Hotamisligil GS, Bischoff J \& Cataltepe S Q2016ख: Dual role of fatty acid-binding protein 5 on endothelial cell fate: a potential link between lipid metabolism and angiogenic responses. Angiogenesis 19 . 
65. Yu S, Levi L, Siegel R \& Noy N囚2012囚: Retinoic acid induces neurogenesis by activating both retinoic acid receptors (RARs) and peroxisome proliferator-activated receptor $\beta / \delta$ (PPAR $\beta / \delta)$. J Biol Chem 287: 42195-42205.

66. Yun-feng LU, Ji-bao C, Bo Z, Qing-gang LI, Zhi-xiu W, Hao Z \& Ke-liang WUヌ2017区: Cloning, expression, and polymorphism of the ECl1 gene in various pig breeds. Journal of Integrative Agriculture 16: 1789-1799.

67. Zhang M, Yan FB, Li F, Jiang KR, Li DH, Han RL, Li ZJ, Jiang RR, Liu XJ, Kang XT \& Sun GR囚2017囚: Genome-wide DNA methylation profiles reveal novel candidate genes associated with meat quality at different age stages in hens. Sci Rep 7: 45564.

68. Zhang Y, Wang Y, Wang X, Ji Y, Cheng S, Wang M, Zhang C, Yu X, Zhao R, Zhang W, Jin J, Li T, Zuo Q \& Li B囚2018『: Acetyl-coenzyme A acyltransferase 2 promote the differentiation of sheep precursor adipocytes into adipocytes. J Cell Biochem .

69. Zhao G P, Chen J L, Zheng M Q, Wen J \& Zhang Y区2007®: Correlated responses to selection for increased intramuscular fat in a Chinese quality chicken line. Poult Sci 86.

70. Zhichao X, Yuting L, Guiying W, Changrong G, Guanghong Z, Wangang Z \& Guozhou L囚2018囚: 1 H NMR-based water-soluble lower molecule characterization and fatty acid composition of boiled Wuding chicken during processing. J Sci Food Agric .

71. Chen Gangyao, Tang Zhigang, Wen Chao, Yang Weibing and Zhou Yanmin (2012): Effect of oligomeric chitosan on antioxidant performance, lipid metabolism and muscle quality of growing and finishing pigs. Food and feed industry: 54-57.

72. Liu Chen. A preliminary study on the biochemical and molecular mechanism of the tissue differences in chicken muscle fat and inosine content: Chinese Academy of Agricultural Sciences, 2009.

73. Chen Jilan, Wen Jie, Wang Shubai, Zhao Guiping, Zheng Maiqing and Li Xiaohua (2005): Research on the deposition of chicken inosine and intramuscular fat. Journal of Animal Science and Veterinary Medicine: 843-845.

74. Chen Jilan, Wen Jie, Zhao Guiping, Zheng Maiqing and Yang Ning (2005): Estimation of genetic parameters of meat flavor traits such as chicken inosine and intramuscular fat. Heredity: 42-46.

75. Dou Tengfei, Wang Shanrong, Liu Lixian, Hu Wenyuan, Tong Huiquan, Gu Dahai, Li Qihua, Jia Junjing, Ge Changrong and Xu Zhiqiang (2016): Correlation between GS gene expression and muscle glycogen content and pH in Wuding chicken and Avian broiler Research. Chinese Poultry 38: 17-20.

76. Li Huifang and Chen Kuanwei (2004): Comparison of muscle inosine and fatty acid content in different chicken breeds. Journal of Yangzhou University: 9-11+32.

77. Li Wufeng, Xu Shangzhong, Cao Honghe and Li Hongbin (2004): Correlation analysis of genetic variation and meat quality traits of the second intron of H-FABP gene in three hybrid cattle breeds. Journal of Animal Science and Veterinary Medicine: 252-255.

78. Lin Yaqiu, Xu Yaou, Zhang Runfeng, Zhang Ming, Zuo Bin and Zheng Yucai (2015): Timing expression of Chemerin and ChemR23 genes in Tibetan chicken and their correlation with intramuscular fat content. Journal of Animal Science and Veterinary Medicine 46: 1290-1299. 
79. Yue Ying. Research on liver proteomics of broilers with different genotypes: Chinese Academy of Agricultural Sciences, 2013.

80. Liu Haifeng, Yan Zhenxin, Dong Han and Li Xuewei (2010): PPARy, DECR1 and ECHS1 gene expression patterns during differentiation of porcine preadipocytes. Journal of Sichuan Agricultural University 28: 215-218.

81. Liu Rong, Ju Yanfang, Yang Jinju, Du Xuemei, Chen Yong, Liu Ying, Gao Jianen and Sun Qihong (2008): Preparation and identification of monoclonal antibodies to enoyl-CoA hydrolase. Journal of Cellular and Molecular Immunology: 387-388+392.

82. Lu Yunfeng, Li Qinggang, Wang Zhixiu, Zhang Bo, Wu Keliang and Zhang Hao (2015): Polymorphism of ECl1 gene in Huai pig breeds and its correlation with back fat thickness. Chinese Journal of Animal Science 51: 1-4 .

83. Lu Zhaoning, Wang Wei and Yan Jingbin (2013): the regulatory mechanism of long-chain acyl CoA dehydrogenase. Journal of medical molecular biology 10: 172-176

84. Luan Yihong, Lu Ning, Wang Jiwei and Yi Qing (2003): biochemical function and application of coenzyme A. Chinese Journal of biochemical medicine 24: 159-161

85. Pan Zhixiong, Lu Jia, Lu Lizhi and Wang Jiwen (2010): cloning of goose ACSL1 gene and its role in the formation of goose fatty liver. Journal of animal husbandry and veterinary medicine 41: 14071413

86. Yu Jiahui. Relationship between E-FABP gene polymorphism and related traits in Jinghai Yellow Chicken: Yangzhou University, 2007

87. Wang Qianqian, Yang Biao, Xia Lili, Sun Xiaoxian, Geng Tuoyu and Gong Daoqing (2016): Cloning of goose acetyl-CoA acyltransferase 2 gene and its expression changes in the formation of goose fatty liver. Journal of Animal Husbandry and Veterinary Medicine 47: 700-708.

88. Wang Qin (2019): Research progress on factors affecting muscle fat content in poultry and related genes. Poultry Science: $53-58$.

89. Li Peng. Characteristics and regulation of liver fatty acid oxidative metabolism in ketosis cows: Jilin University, 2012.

90. Li Wenjuan. Screening of Chicken Fat Quality Related Genes for Fat Metabolism and Nutritional Regulation: Chinese Academy of Agricultural Sciences, 2008

91. Jiang Ruirui. The effect and mechanism of niacin on chicken fat metabolism and meat quality: Chinese Academy of Agricultural Sciences, 2010.

92. Zhang Guangmin. The effect of conjugated linoleic acid on the quality of meat and chicken meat and its mechanism of action: Chinese Academy of Agricultural Sciences, 2006.

93. Wang Shubai. Chicken Inosinate Deposition and Nutritional Regulation: Chinese Academy of Agricultural Sciences, 2004.

94. Yu Mengmeng, Li Meng, Li Shanshan, et al. Effect of Fatty Acid Binding Protein 5 on Milk Fat Synthesis of Dairy Cow Mammary Epithelial Cells. Chinese Journal of Animal Husbandry and 
Veterinary Medicine, 2017,44(04):941-949.

95. Zang Shasha, Liu Yixuan, Song Guangyao and Wang Chao (2014): The effect of Jinlida on skeletal muscle lipid deposition and mitochondrial function in insulin resistant rats. Chinese Patent Medicine 36: 1371-1376.

96. Zhang Qingqiu, Shi Hui, Wang Yuxiang, Li Hui and Wang Qigui (2012): Effects of changes in A-FABP gene expression in chicken preadipocytes on PPARy, perilipin and E-FABP expression. Journal of Animal Science and Veterinary Medicine 43: 1531-1538.

97. Zhou Guoli, Zhu Qi, Guo Shanli and Wu Yuhou (2005): Analysis of the polymorphism of Luxi cattle HFABP gene and its relationship with meat quality traits. Northwest Agricultural Journal: 10-12.

\section{Tables}

Due to technical limitations, table 1 is only available as a download in the Supplemental Files section.

\section{Figures}
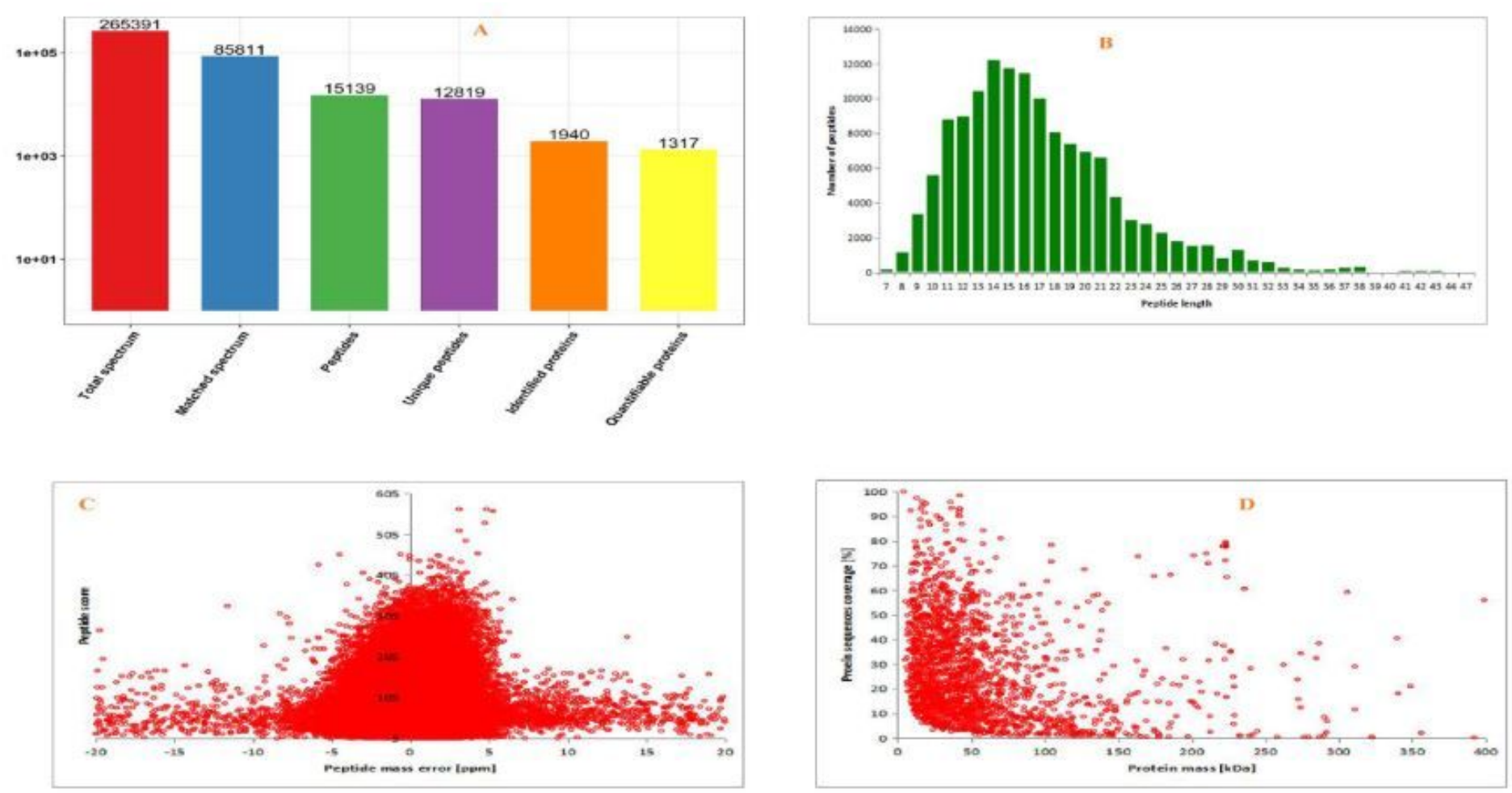

\section{Figure 1}

The results of label-free proteomics on protein identification of Jingyuan chicken leg and pectoral muscle tissues; A: Basic statistics of mass spectrometry data results; B: Distribution of peptide sequence length after digestion of the identified proteins; $\mathrm{C}$ : The molecular mass error distribution diagram of the identified protein peptides; $\mathrm{D}$ : The mass distribution diagram of the identified protein molecules. Note: TJ stands for leg muscles and XJ stands for pectoral muscles. 

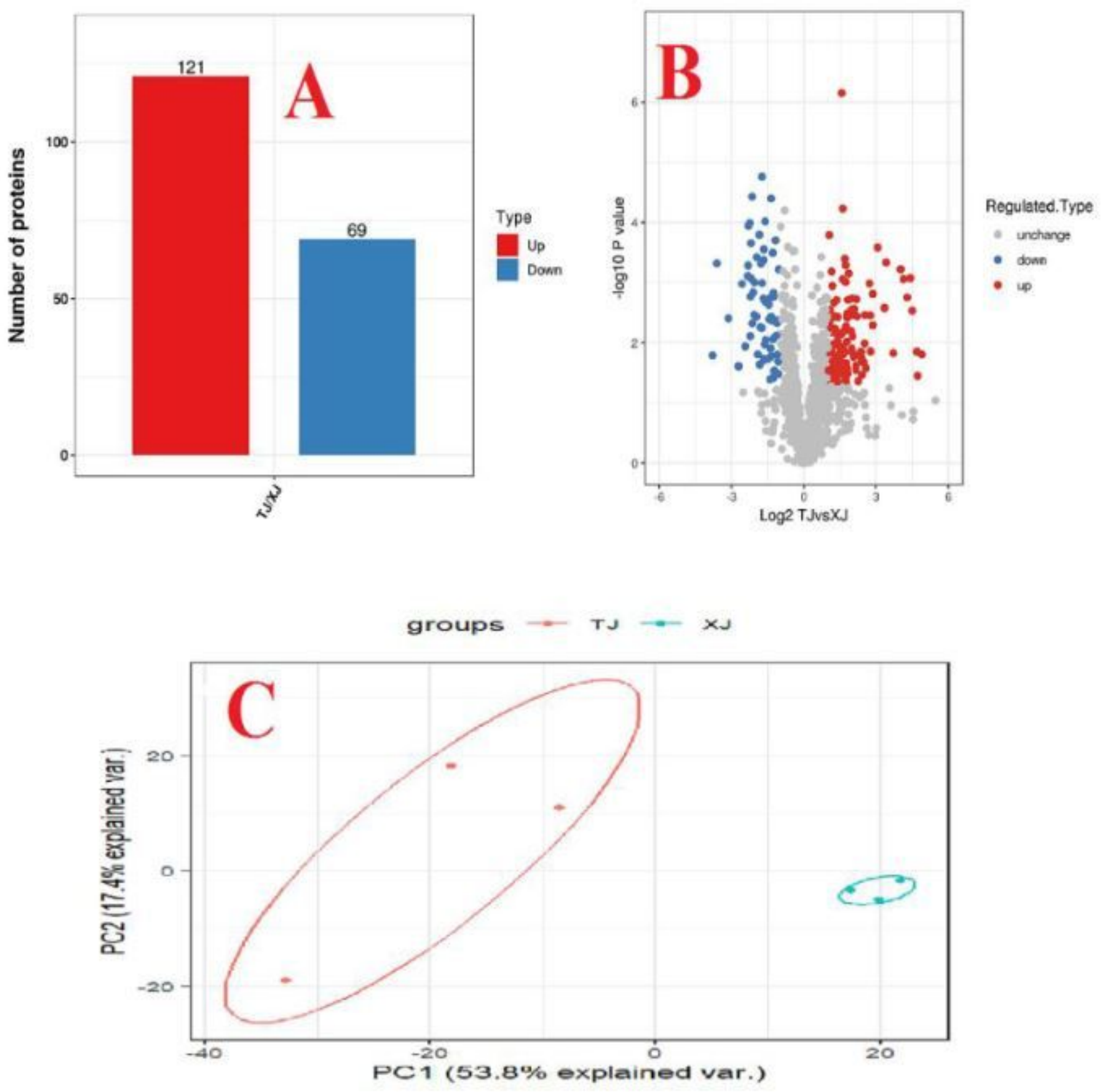

Figure 2

Statistical analysis of differentially expressed proteins of Jingyuan chicken leg muscles and pectoral muscles; A: Statistical analysis of differentially expressed proteins of Jingyuan chicken leg muscles and pectoral muscles; B: Quantitative volcano graph of differentially expressed proteins of Jingyuan chicken leg muscles and pectoral muscles; $\mathrm{C}$ : Two dimensional scatter chart of protein quantitative main component analysis (PCA) between repeated samples of Jingyuan chicken leg muscle and chest muscle. Note: TJ stands for leg muscles and XJ stands for pectoral muscles. 


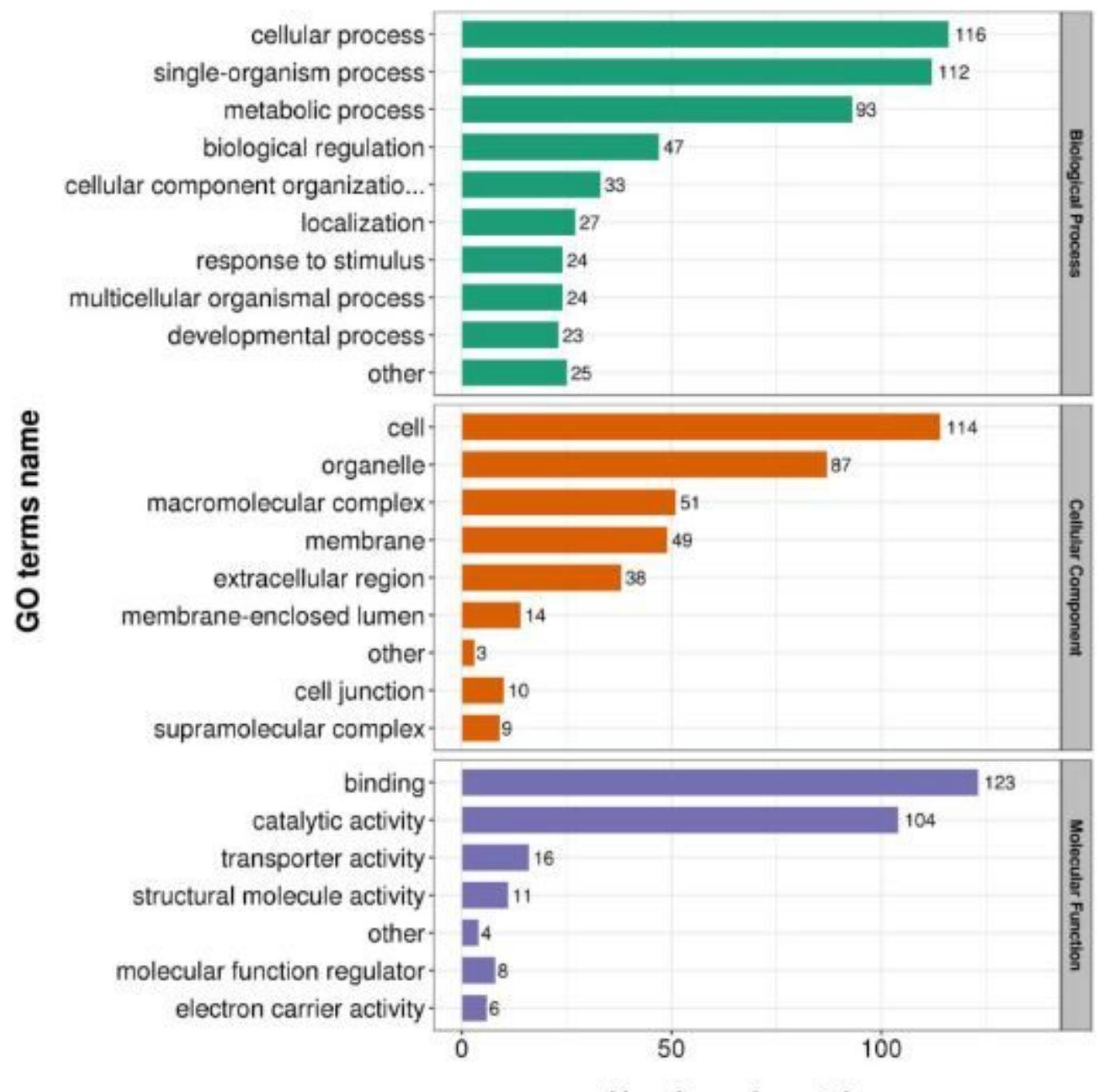

Number of proteins

\section{Figure 3}

Statistical distribution of Jingyuan chicken leg muscle and pectoral muscle differentially expressed proteins in GO secondary classification. Note: TJ stands for leg muscles and XJ stands for pectoral muscles. 


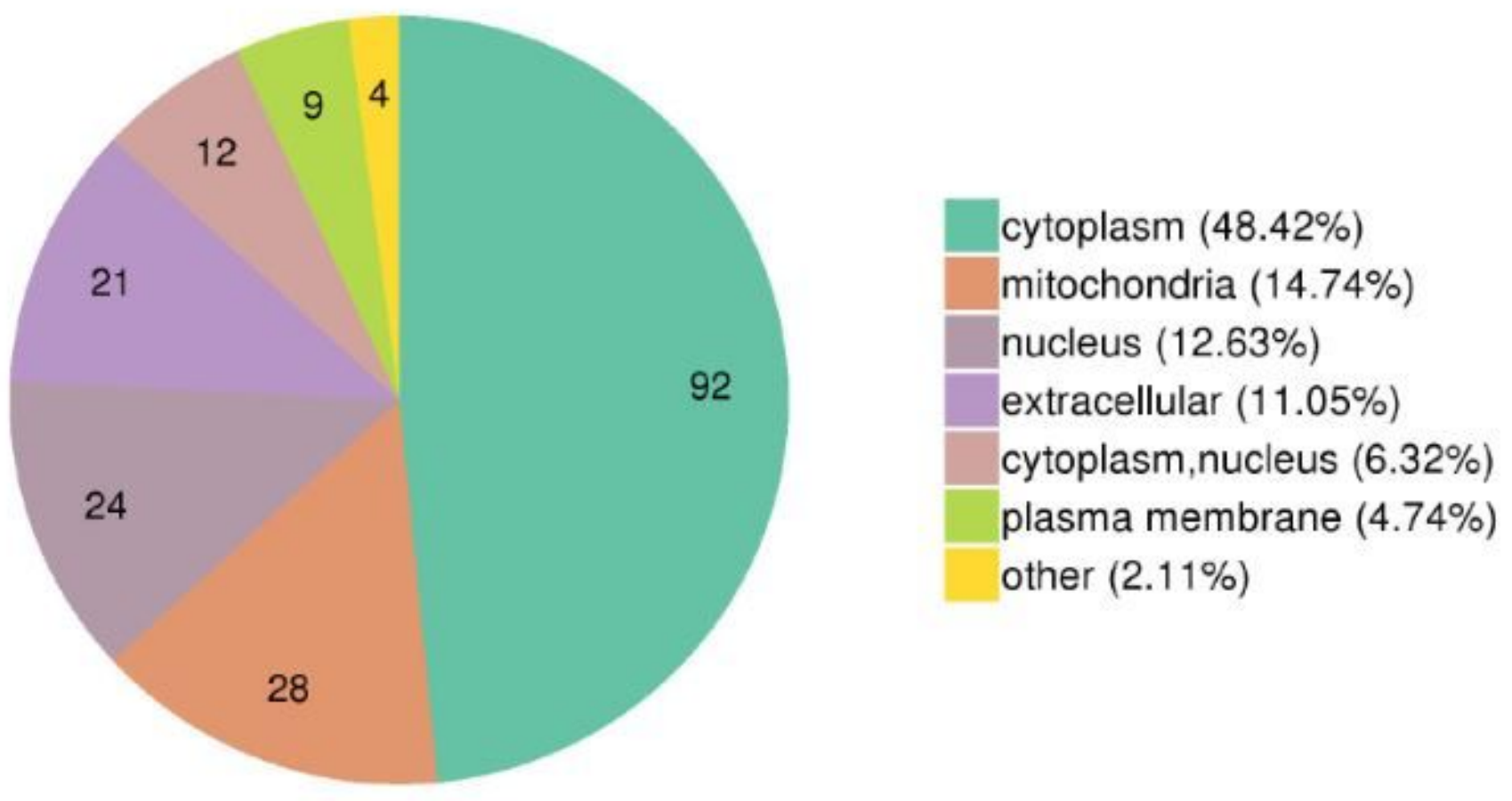

Figure 4

Location map of differentially expressed proteins of Jingyuan chicken leg muscle and pectoral muscle in subcellular structure. Note: TJ stands for leg muscles and XJ stands for pectoral muscles. 


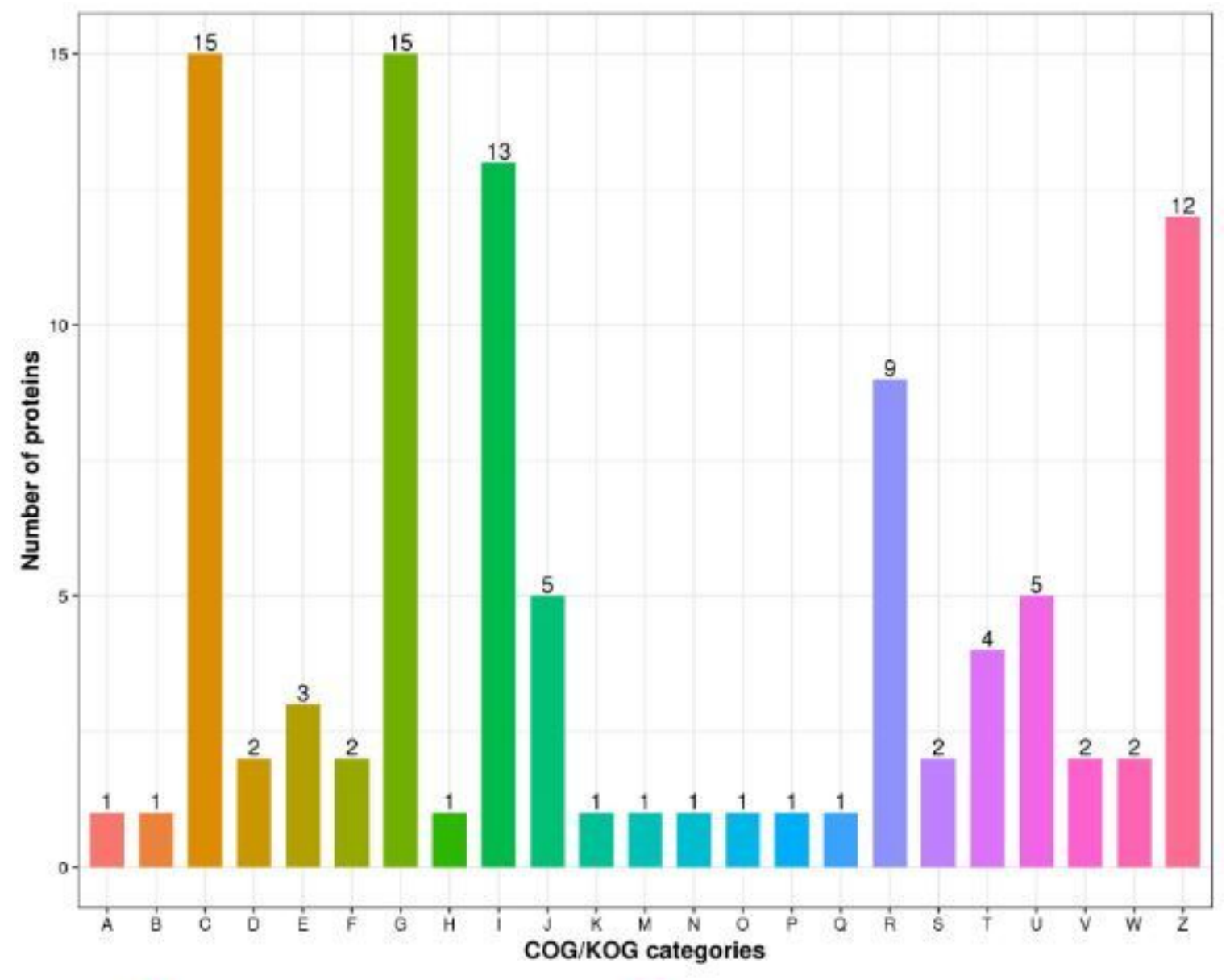

[A] RNA procassing and modification
[B] Chromafin structurs and dynamics
[C] Energy produstion and conversion
[D] Cell cycle control, cell division, chromosome partitioning
[E] Amino acid transport and metabolism
[F] Nucleotde transport and metabolism
[G] Carbohydrabe transpart and metabolism
[H] Coenzyme transport and mataboism
[] Lipid transport and metabolism
[J] Translation, ribosomal structure and biogenesis
[K] Transcripion
[M] Cell wallmembraneienvelopo biogonesis

[N] Cell motility
[O] Postranalational modification, protain turnover, chaperones
[P] Inorganic ion transport and metabolism
[O] Secondary metabolites biosynthesis, transport and catabolism
[P] General function prediction onty
[S] Function unknown
[T] Signal transduction mechanisms
[U] Intracellular trafficking, secrotion, and vesicular transport
[V] Defense mechanisms
[W] Extracellular structures
[Z] Cytaskeleton

$[$ [Z] Cybskeleton

\section{Figure 5}

COG function classification and distribution of differentially expressed proteins in Jingyuan chicken leg muscle and pectoral muscle. Note: TJ stands for leg muscles and XJ stands for pectoral muscles. 


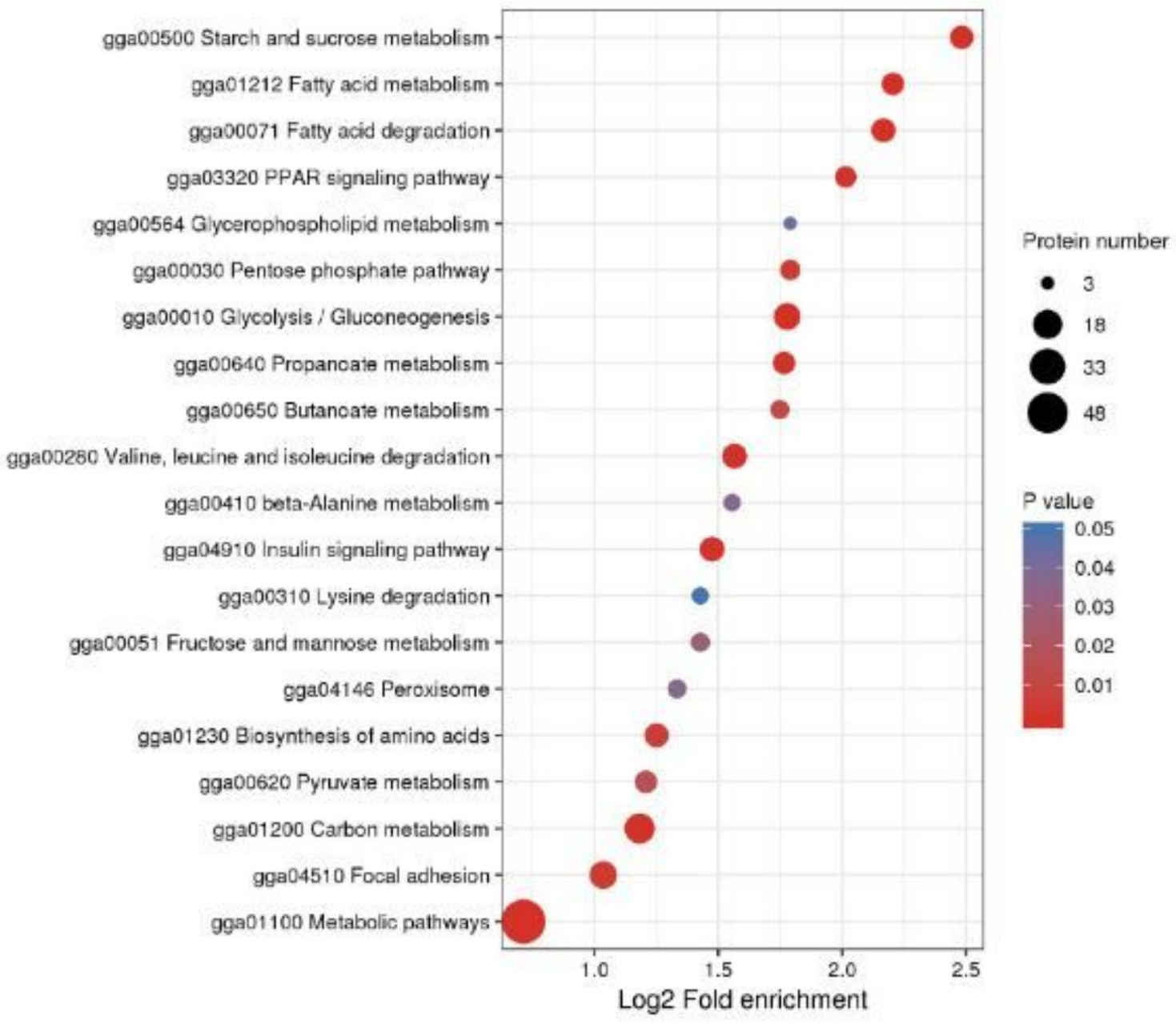

\section{Figure 6}

Enrichment and distribution map of differentially expressed proteins of Jingyuan chicken leg muscle and pectoral muscle in GO functional classification. Note: TJ stands for leg muscles and XJ stands for pectoral muscles. 


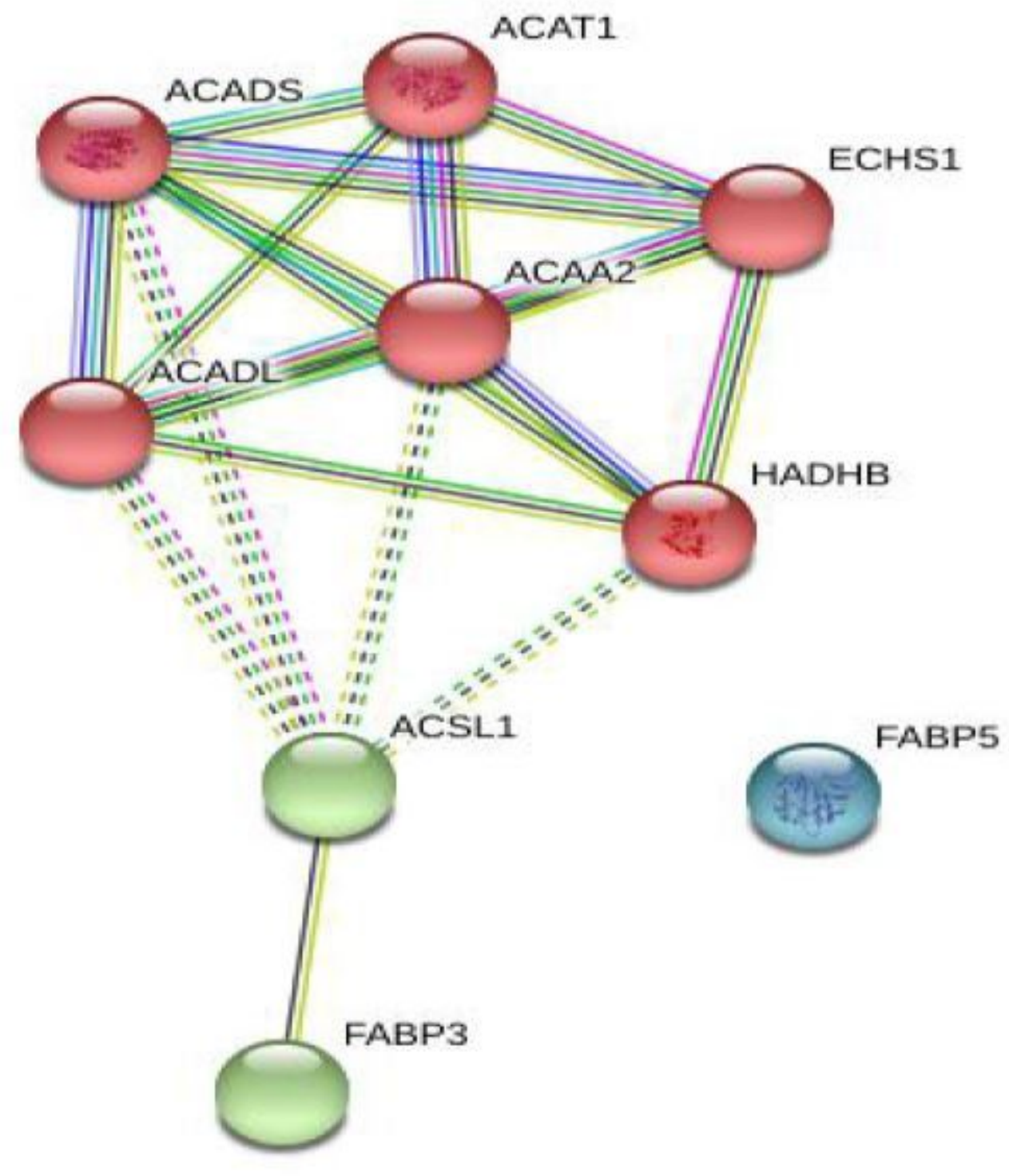

Figure 7

Network interaction path diagram of differentially expressed proteins of Jingyuan chicken leg muscle and pectoral muscle. Note: TJ stands for leg muscles and XJ stands for pectoral muscles.

\section{Supplementary Files}

This is a list of supplementary files associated with this preprint. Click to download.

- Table1.JPG 